\title{
DISPLAYING STATISTICAL POINT ESTIMATORS: THE LEADING-DIGIT PROCEDURE
}

\author{
Wheyming T. Song \\ Department of Industrial Engineering \\ National Tsing Hua University \\ Hsinchu, Taiwan, 300 \\ Republic of China \\ wheyming@ie.nthu.edu.tw
}

\author{
Bruce W. Schmeiser \\ School of Industrial Engineering \\ Purdue University \\ West Lafayette, IN 47907-2023, U.S.A. \\ bruce@ecn.purdue.edu
}

\begin{abstract}
We propose a procedure for reporting a statistical point estimator and its precision for statistical experiments such as simulation experiments. Based on three criteria - loss of statistical information, number of characters required, and likelihood of user misinterpretation-we advocate our procedure for use when reporting many point estimators in tabular form. The procedure discards meaningless digits of the point estimator, and all but the left-most non-zero digit of the standard error. These two resulting values are separated by the “;” sign.
\end{abstract}

\section{Introduction}

Statistical experiments such as simulation experiments produce data from which one or more point estimators are computed. Such a point estimator, a random variable that is denoted here by $\widehat{\Theta}$, is used to guess the unknown value of a system performance measure, a constant that is denoted here by $\theta$. Typically the bias of $\widehat{\Theta}$ is negligible compared to its standard error, $\sigma$, the square root of its variance.

Having run such an experiment, the practitioner has a single point-estimator observation, denoted by $\widehat{\theta}$. Its value is known only to some finite number of digits, often determined by an output device associated with the experiment. Typically the number of digits is more than needed to specify $\widehat{\theta}$ to the precision indicated by the standard error $\sigma$, which we assume to be known. (Our recommendations below also hold when $\sigma$ is only estimated.) The motivation of this paper is reporting the point estimate $\widehat{\theta}$ in a way that reflects its precision, as measured by $\sigma$.

The organization of this paper is as follow. We summarize disadvantages of confidence intervals in Section 2, discuss the probabilistic behavior of the leading digit in Section 3, and propose the leading-digit format in Section 4.

\section{Confidence Interval Procedures}

Before discussing our recommended procedure, we briefly mention confidence intervals, the reporting-problem solution available to everyone who has taken in a first course in probability and statistics. A confidence-interval procedure produces a random interval $\left(L_{\alpha}, U_{\alpha}\right)$ that includes $\theta$ with probability $1-\alpha$ whenever the underlying assumptions hold. Song and Schmeiser (1994) discuss six disadvantages of confidence intervals. We summarize them below:

One. Users, especially those with little statistical background, often misinterpret the interval's meaning by forgetting that the CIP probability statement is about the procedure, not about any one observed interval.

Two. Even when the user carefully interprets the interval, violations of the normality and independence assumptions causes the true coverage probability to differ from the nominal value. This difference is the motivation of many research papers (e.g., Schmeiser 1982, Schmeiser 1992, Song and Schmeiser 1993, 1995) that propose and evaluate CIP's for various simulation contexts, especially that of stationary autocrrelated data.

Three. The coverage probability is arbitrary. Few users have a reason for the chosen value of $\alpha$ or sufficient intuition to sense the difference between a $90 \%$ and a $99 \%$ confidence. Conway, Maxwell, McClain and Worona (1987) argue that real-world conclusions should not depend upon details of formal statistics (such as the choice of $\alpha$ ). Goldsman, Nelson and Schmeiser (1991) argue (with each other) about the need for formal methods to compare systems.

Four. Degrees of freedom for dependent data are difficult to specify. Specifically, Degrees of freedom are sometimes well specified, such as when the confidence interval is computed from independent replications or nonoverlapping batch means 
(Schmeiser 1982). But other methods, such as overlapping batch means (Schmeiser and et al 1990), do not approach normality through Student's T distribution.

Five. Stating a result as a confidence interval $\left(L_{\alpha}, U_{\alpha}\right)$ forces the reader to do mental arithmetic to determine the point estimator $\hat{\theta}$, which is the most important number. The standard error, the other fundamental number, is also difficult or impossible to recover.

Six. Finally, but not the least important, is Stating confidence intervals is cumbersome. When simulation experiments are summarized in a table, clarity is greatly aided by stating only $\hat{\theta}$, and then to as few digits as are practically and statistically significant.

Ironically illustrating these six disadvantages, confidence intervals are used by (almost) no researchers in presenting their Monte Carlo results of confidence-interval performance. Most are aware, however, of the need to assess the effect of sampling error on the point estimator. Tables of estimated coverage probabilities corresponding to various data types and CIP's often contain single entries $\hat{\theta}$ or sometimes double entries such as $\hat{\theta} \pm \hat{\sigma}, \theta(\hat{\sigma})$, or with $\hat{\sigma}$ printed below $\hat{\theta}$, where $\hat{\sigma}$ is the estimated $\sigma$.

\section{Statistical Behavior of $\widehat{\Theta}$ and of its Digits}

The purpose of this section is to lay the foundation for arguing for truncating all digits to the right of position $l$, the leading digit of $\sigma$. The foundation is based on the loss of statistical information in the point estimate $\widehat{\Theta}$ caused by truncating to $\widehat{\Theta}_{j}$, as a function of $j$.

\subsection{Notation and Definitions}

The decision of which digits of the (observed) point estimate $\widehat{\theta}$ to report involves the (unknown constant) performancemeasure value $\theta$, the distribution of the (random) point estimator $\widehat{\Theta}$, the (observed) point estimate $\widehat{\theta}$, and the (known constant) standard error $\sigma$.

For every integer $j$, let $t_{j}$ denote the $j$ th digit of the performance measure $\theta$ and let $\theta_{j}$ denote the value of $\theta$ truncated to the $j$ th position; that is,

$$
|\theta|=\cdots t_{2} t_{1} t_{0} \cdot t_{-1} t_{-2} \cdots=\sum_{j=-\infty}^{\infty} t_{j} 10^{j}
$$

and

$$
\left|\theta_{j}\right|=\left\lfloor|\theta| / 10^{j}\right\rfloor \times 10^{j}=\ldots t_{j+2} t_{j+1} t_{j} \times 10^{j}
$$

where absolute values are used to ignore the sign of $\theta$. To avoid ambiguity, when $\theta$ has two real-number representa- tions (such as $\theta=-1=-0 . \overline{999}$ ), define $\theta_{j}$ and $t_{j}$ using the finite-digit representation.

Analogous to $\theta_{j}$ and $t_{j}$ for $\theta$, define $\widehat{\theta}_{j}$ and $\widehat{t}_{j}$ for the point estimate $\widehat{\theta}$ and define $\widehat{\Theta}_{j}$ and $\widehat{T}_{j}$ for the point estimator $\widehat{\Theta}$. That is,

$$
|\widehat{\boldsymbol{\theta}}|=\cdots \hat{t}_{2} \hat{t}_{1} \hat{t} 0 . \hat{t}_{-1} t_{-2} \cdots=\sum_{j=-\infty}^{\infty} \hat{t}_{j} 10^{j}
$$

and

$$
\left|\widehat{\theta}_{j}\right|=\left\lfloor|\theta| / 10^{j}\right\rfloor \times 10^{j}=\ldots \hat{t}_{j+2} \hat{t}_{j+1} \hat{t}_{j} \times 10^{j}
$$

Analogously, let $s_{j}$ denote the $j$ th digit of $\sigma$ and let $l$ denote the digit position of the leading digit of $\sigma$; then the leading digit is $s_{l}$. If $\sigma=0$, then define $r=-\infty$, corresponding to the limiting standard-error value as sampling continues forever. Let $\sigma_{j}$ denote $\sigma$ truncated at the $j$ th position. Then

$$
\sigma_{j}=\left\lfloor\sigma / 10^{j}\right\rfloor \times 10^{j},
$$

the value of the standard error truncated to the digits $\cdots s_{j+1} s_{j}$.

\subsection{Distributions of the truncated point estimator $\widehat{\Theta}_{j}$}

We could measure the quality of the point estimator $\widehat{\Theta}$ truncated at the $j$ th digit using

$$
\mathrm{P}\left(\left|\widehat{\Theta}_{j}-\theta_{j}\right| \leq h \times 10^{j}\right)
$$

for any $h=0,1, \ldots$. That is, the point estimator succeeds if its truncated value is within $h$ units of the truncated value of the true value $\theta$. A bit arbitrarily, we choose to use $h=0$, exact equality, yielding a specific definition of the probability of correct truncation.

Before discussing the probability of correct truncation, we want to discuss two distributions associated with $\widehat{\Theta}_{j}$. First, define

$$
p_{j}(i) \equiv \mathrm{P}\left(\ldots, \widehat{T}_{j+2}=t_{j+2}, \widehat{T}_{j+1}=t_{j+1}, \widehat{T}_{j}=i\right)
$$

for $i=0,1, \ldots, 9$, the joint probability that all digits to the left of column $j$ are correct and that $\hat{T}_{j}$, the digit in column $j$, takes the specified value $i$.

Second, we define the conditional distribution of $\hat{T}_{j}$ given that all previous digits are correct. Let

$$
c_{j}(i) \equiv \mathrm{P}\left(\widehat{T}_{j}=i \mid \widehat{\Theta}_{j+1}=\theta_{j+1}\right)
$$

for $i=0,1, \ldots, 9$, the conditional probability that $\widehat{\Delta}_{j}=i$ given that all previous digits are correct. For every integer 
value of $j$, these probabilities are related by

$$
c_{j}(i)=p_{j}(i) / \sum_{k=0}^{9} p_{j}(k)
$$

for $i=0,1, \ldots, 9$ and therefore $\sum_{i=0}^{9} c_{j}(i)=1$.

\subsection{Measuring the quality of $\widehat{\Theta}_{j}$}

The "correct-truncation" probability of the point estimator $\widehat{\Theta}$ truncated at the $j$ th digit is

$$
p_{j} \equiv \mathrm{P}\left(\ldots, \widehat{T}_{j+2}=t_{j+2}, \widehat{T}_{j+1}=t_{j+1}, \widehat{T}_{j}=t_{j}\right)=p_{j}\left(t_{j}\right)
$$

the joint probability that all digits of $\widehat{\Theta}$ are correct through position $j$.

As discussed in the next subsection, these joint probabilities can be used to compute the "meaningful-digit" probability

$c_{j} \equiv \mathrm{P}\left(\widehat{\Theta}_{j}=\theta_{j} \mid \widehat{\Theta}_{j+1}=\theta_{j+1}\right)=\mathrm{P}\left(\widehat{T}_{j}=t_{j} \mid \widehat{\Theta}_{j+1}=\theta_{j+1}\right) \equiv c_{j}\left(t_{j}\right)$,

the probability that the $j$ th digit of $\widehat{\Theta}$ is correct conditional that all previous digits are correct.

\subsection{Properties}

Given values of the performance measure $\theta$ and the standard error $\sigma$, computation of $p_{j}$ and $c_{j}$ is straightforward. In particular, the correct-digit probability is

$$
c_{j}=p_{j} / p_{j+1}
$$

and the correct-truncation probability is

$$
p_{j}= \begin{cases}\mathrm{P}\left(0 \leq \widehat{\Theta}-\theta_{j}<10^{j}\right), & \theta \geq 0 \\ \mathrm{P}\left(-10^{j}<\widehat{\Theta}-\theta_{j} \leq 0\right), & \theta<0 .\end{cases}
$$

Because of the discreteness of truncation, the detailed behavior of $p_{j}$ and $c_{j}$ as a function of $\theta, \sigma$, and $j$ is complex. The general behavior of the truncation position $j$, however, does not depend upon $\theta$ and $\sigma$. Begin with large values of $j$, corresponding to substantial truncation. The values of $p_{j}$ are close to one. Substantial truncation guarantees correctness, in a trivial sense, because

$$
\lim _{j \rightarrow \infty} p_{j}=1,
$$

due to the left-most digits of both $\widehat{\Theta}_{j}$ and $\theta$ being zero. At the other extreme,

$$
\lim _{j \rightarrow-\infty} p_{j}=0
$$

because the right-most digits $\widehat{T}_{j}$ are independent of each other. Between these two extremes, the infinite and continuous range of $\widehat{\Theta}$ implies that $0<c_{j}<1$ for every position $j$; therefore the sequence $p_{j}$ is monotonically decreasing, i.e.,

$$
1>\cdots>p_{2}>p_{1}>p_{0}>p_{-1}>\cdots>0 .
$$

For asymptotically small values of $j$, the $p_{j}(i)$ values are easy to approximate, because

$$
\lim _{j \rightarrow-\infty} \frac{p_{j}(i)}{f(\theta) 10^{j}}=1
$$

where $f$ denotes the density function of $\widehat{\Theta}$. This limiting value provides a good approximation for all $p_{j}(i)$ for $j<l$ (that is, in positions to the right of the leading-digit position l). (For the normal distribution, $f(\theta)=(\sigma \sqrt{2 \pi})^{-1} \approx(\sigma \times$ 2.507 $)^{-1}$.)

For $i=0,1, \ldots, 9$, the asymptotic result in Equation (5) leads to

$$
\lim _{j \rightarrow-\infty} c_{j}(i)=\lim _{j \rightarrow-\infty} \frac{p_{j}(i)}{p_{j+1}}=\lim _{j \rightarrow-\infty} \frac{p_{j}}{p_{j+1}}=0.1 .
$$

That is, the right-most digits of $\widehat{\Theta}$ are uniformly distributed and therefore meaningless.

\subsection{Probability bounds}

Here we provide probability bounds for $p_{l}, p_{l-1}$ and $c_{l-1}$ in the experiment when $10^{l} \leq \sigma<10^{l+1}$, where $l$ is the rightmost reported position, and $l-1$ is the left-most unreported position. These bounds are the basis for arguing in Section 2.2 that the unreported digit in the Position $l-1$ contains negligible statistical information. The derivation of these bounds in given in Song and Schmeiser (2007).

Result 1 (Song and Schmesier 2007). If $\widehat{\Theta}$ is normally distributed, then for any values of the performance measure $\theta$ and standard error $10^{l} \leq \sigma<10^{l+1}$ (i.e., with leading digit in Position $l$ ),

$$
p_{l} \geq \Phi\left(10^{l} / \sigma\right)-0.5
$$

Therefore, always

$$
p_{l} \geq \Phi(1 / 10)-0.5 \approx 0.0398,
$$


with equality in the limit as $\sigma \rightarrow 10^{l+1}$. When $\sigma=10^{l}$,

$$
p_{l} \geq \Phi(1)-0.5 \approx 0.3413
$$

with equality when $\theta_{l}=\theta$.

Although primarily interesting for $p_{l}$, the monotonicity of the $p_{j}$ values implies that the bounds also hold for all $j>l$.

Similarly, the probability that $\widehat{\Theta}$ is correct through the first unreported digit $\widehat{\Delta}_{l-1}$ has a tight upper bound.

Result 2 (Song and Schmesier 2007). If $\widehat{\Theta}$ is normally distributed, then for any values of the performance measure $\theta$ and standard error $10^{l} \leq \sigma<10^{l+1}$ (i.e., with leading digit in Position $l$ ),

$$
p_{l-1} \leq 2\left[\Phi\left(10^{l-1} /(2 \sigma)\right)-0.5\right] \text {. }
$$

Therefore, $\sigma=10^{l}$ yields the minimal upper bound

$$
p_{l-1} \leq 2[\Phi(1 / 20)-0.5] \approx 0.0399 .
$$

Although primarily interesting for $p_{l-1}$, the monotonicity of the $p_{j}$ values implies that the bounds also hold for all $j<l-1$.

Result 3 (Song and Schmesier 2007). If $\widehat{\Theta}$ is normally distributed, then for any values of the performance measure $\theta$ and standard error $10^{l} \leq \sigma<10^{l+1}$ (i.e., with leading digit in Position $l$ ), then

$$
c_{l-1} \leq \frac{2\left[\Phi\left(10^{l-1} /(2 \sigma)\right)-0.5\right]}{\Phi\left(10^{l} / \sigma\right)-0.5} .
$$

Therefore, $\sigma=10^{l}$ yields the minimal upper bound

$$
\begin{aligned}
c_{l-1} & \leq \frac{2[\Phi(1 / 20)-0.5]}{\Phi(1)-0.5} \\
& \approx 0.117 .
\end{aligned}
$$

Although primarily interesting for $c_{l-1}$, the monotonicity of the $c_{j}$ values implies that the bounds also hold for all $j<l-1$.

These bounds are used in Section 4 to argue that the leading-digit logic provides a reasonable rule for determining when to report a new digit of $\widehat{\theta}$.

\section{Recommended Reporting Procedure}

Taken together, solutions to the three specific problems form a procedure for converting the practitioner's point estimate $\widehat{\theta}$ and standard error $\sigma$ to a string of characters to be printed in a report.

The preferred procedure is application specific, as well as subject to the practitioner's preferences. Nevertheless, we recommend here a procedure that is intended to be widely applicable and appealing. We are guided by three criteria:
(1) loss of statistical information, (2) number of printed characters, and (3) likelihood of user misinterpretation. The first and third criteria have been measured only by our subjective judgment, often aided by interesting conversations with various helpful colleagues.

\subsection{The ; Format}

The approach of combining precision information with the point estimate while maintaining readability was proposed in Song and Schmeiser (1994) and Yoneda (1996). In this paper, we propose a leading-digit format: $\widehat{\theta}_{l}$ and $\sigma_{l}$. That is, the point estimator is truncated to position $l$ and the standard error is truncated to the leading digit. A character string displays these two values separated by the ";", (pronounced as the word $a t$ ) sign. The magnitude of the point estimator is indicated with an explicit decimal point, with an implicit decimal point, with the character " $\mathrm{X}$ " as a place holder, or with scientific notation. The magnitude of the standard error is indicated by the position $l$ of the point-estimator's trailing digit $\hat{l}_{l}$; the value of the standard error is indicated by the digit $s_{l}$. Whether to truncate or round $\widehat{\theta}_{l}$ and $\sigma_{l}$ is a minor decision; we round in our examples and computer code.

\subsection{Examples and Discussion}

Here are some examples of output in the "," format. Suppose that $\widehat{\theta}=123.45678$ and $\sigma=0.011345$. The ";" format produces the character string "123.46;1". The first six characters are $\widehat{\theta}$ without its last three digits. The ";" sign separates the point estimator from its precision indicator, which is $s_{l}=s_{-2}=1$, the leading digit of $\sigma$. The ";" sign is simply a non-numeric separator. The digits $\hat{t}_{-3}=6$, $\hat{t}_{-4}=7, \hat{t}_{-5}=8$ are omitted because they are to the right of the trailing digit $\hat{t}_{l}=\hat{t}_{-2}=5$.

The string of characters needs to be modified for large and small values of $\widehat{\theta}$, when the lack of a decimal point leaves the magnitude ambiguous. For example $\widehat{\theta}=123456.78$ and $\sigma=113.45$ results in the character string $1235 X X ; 1$, where the character $X$ is a place holder. The digits $\hat{t}_{1}=5$ and $\hat{t}_{0}=6$ are not printed because the standard error's value indicates that their values are meaningless. The scientific notation "1235E3;3", with the character $E$ indicating a power of ten, is used for $\widehat{\theta}=1234567.8$ and $\sigma=3113.45$.

Table 1 provides a sequence of examples. The first eight consider $\widehat{\theta}=1234567.8$ with the standard error getting progressively larger. For now we assume that there are no space limitations and that the for $\widehat{\theta}$ and $\sigma$ are specified to infinite precision.

In the first row, the standard error .0000311345 is miniscule compared to the point estimator, with the trailing digit $\hat{t}_{5}=0$ being specified only implicitly. The corresponding "," format contains thirteen digits-seven digits to the left 
Table 1: Examples of the ";" format with infinite-precision specified values and unlimited space available for printing.

\begin{tabular}{crrr} 
example & $\widehat{\theta}$ & $\sigma$ & “;” Format \\
\hline 1 & 1234567.8 & .0000311345 & $1234567.80000 ; 3$ \\
2 & 1234567.8 & .00311345 & $1234567.800 ; 3$ \\
3 & 1234567.8 & .311345 & $1234567.8 ; 3$ \\
4 & 1234567.8 & 31.1345 & $123457 X ; 3$ \\
5 & 1234567.8 & 3113.45 & $1235 E 3 ; 3$ \\
6 & 1234567.8 & 311345. & $12 E 5 ; 3$ \\
7 & 1234567.8 & $311345 E 2$ & $0 E 7 ; 3$ \\
8 & 1234567.8 & $311345 E 4$ & $0 E 9 ; 3$ \\
\hline
\end{tabular}

of the decimal point and five to the right. The final digit of $1234567.80000 ; 3$ is determined by $s_{l}=s_{-5}=3$, which is the last character printed.

The progression through the next five examples is straight forward, but the seventh and eighth examples require comment because their ";" format contains no digits of $\widehat{\theta}$. The standard errors are more than 25 and 2500 times bigger than the point estimator, respectively, so in both examples the point estimator is far from statistically different from zero; all digits are meaningless. Therefore, the point-estimator value printed is zero.

These two examples also illustrate the careful interpretation of the leading standard-error digit $s_{l}=3$. The only reason to show a magnitude for zero $(0 E 7 ; 3$ and $0 E 9 ; 3)$ is to indicate the value of the standard error, which is always given by the position of the trailing digit of $\widehat{\theta}$.

Table 2 contains the same examples as Table 1, except that now the specified value of $\widehat{\theta}$ is assumed to contain only seven accurate digits, as would arise from 32-bit floatingpoint computing. The number of digits printed is now the minimum of seven and the number allowed by the standard error (in Table 1).

Only examples 1-3 are affected by the restriction on the number of available digits. These three examples illustrate another point about interpreting the standard-error shown, which is zero. Although the leading digit is $s_{l}=3$, to print a three would be misleading because the standard error's magnitude is no longer indicated by the trailing digit of $\widehat{\theta}$. Therefore a zero appears after the ";" sign, with the interpretation that the standard-error digit corresponding to $\hat{t}_{0}=8$ is $s_{0}=0$.

To be specific, the printed standard-error digit always corresponds to the trailing digit of $\hat{\theta}$, even when the trailing digit is not $\hat{t}_{l}$. In these cases the standard error's value is known only to be substantially less than required to ensure that the trailing digit is known with negligible sampling error.
Table 2: Examples of the ";" format with single-precision specified values and unlimited space available for printing.

\begin{tabular}{crrr} 
example & $\widehat{\theta}$ & $\sigma$ & “"” Format \\
\hline 1 & 1234567.8 & .0000311345 & $1234568 ; 0$ \\
2 & 1234567.8 & .00311345 & $1234568 ; 0$ \\
3 & 1234567.8 & .311345 & $1234568 ; 0$ \\
4 & 1234567.8 & 31.1345 & $123457 X ; 3$ \\
5 & 1234567.8 & 3113.45 & $1235 E 3 ; 3$ \\
6 & 1234567.8 & 311345. & $12 E 5 ; 3$ \\
7 & 1234567.8 & $311345 E 2$ & $0 E 7 ; 3$ \\
8 & 1234567.8 & $311345 E 4$ & $0 E 9 ; 3$ \\
\hline
\end{tabular}

Table 3: Examples of the ";" format with single-precision specified values and space for only eight characters.

\begin{tabular}{crrr} 
example & $\widehat{\theta}$ & $\sigma$ & “; Format \\
\hline 1 & 1234567.8 & .0000311345 & $1235 E 3 ; 0$ \\
2 & 1234567.8 & .00311345 & $1235 E 3 ; 0$ \\
3 & 1234567.8 & .311345 & $1235 E 3 ; 0$ \\
4 & 1234567.8 & 31.1345 & $1235 E 3 ; 0$ \\
5 & 1234567.8 & 3113.45 & $1235 E 3 ; 3$ \\
6 & 1234567.8 & 311345. & $12 E 5 ; 3$ \\
7 & 1234567.8 & $311345 E 2$ & $0 E 7 ; 3$ \\
8 & 1234567.8 & $311345 E 4$ & $0 E 9 ; 3$ \\
\hline
\end{tabular}

Table 3 again contains the same eight examples. A third restriction - space - is added to the number of digits printed. In Table 2, the first four examples took nine characters. When tabling results in simulation output or summarizing statistical data in general, limiting results to a fixed number of characters simplifies the presentation of results.

In Table 3 we require that no more than eight characters are used in the ";" format, changing the results for Examples 1-4. Two characters are required to specify the precision, leaving only six characters for $\hat{\theta}$, which is expressed as $1235 E 3$. Here again the ; 0 means that the trailing digit is known with negligible sampling error.

An alternative to the ";" format when the standard-error digit is zero is to omit; 0 , thereby freeing all eight characters to specify $\widehat{\theta}$. Examples $1-3$ would then produce 1234568 . with the interpretation that $\hat{t}_{0}=8$ (after rounding) is known with negligible sampling error. Example 4 would produce $123457 X X$ but the of value $\hat{t}_{6}$ has substantial sampling error, although the eight-character ";" format has ;0. For consistency across examples, we have chosen to always show the standard-error information in the last two characters. 


\section{3 "; Format" Procedure}

The "; format" procedure is as follows. Four values are input: the point estimate $\widehat{\theta}$, the standard error $\sigma$, the number of available digits $n$, and the maximum number of printed characters $m$. The procedure returns a string of $m$ characters. (In practice, our code also returns an error indicator to report invalid input-paramter values.)

1. Record all digits to the left of and including $\hat{t}_{l}$, excluding all leading zeros to the left of $\hat{t}_{l}$ and to the left of the decimal point.

2. Convert the recorded digits to a character string, based on the location of the decimal point.

(a) If the decimal point is to the left of or within the recorded digits, the string is composed of the digits and the decimal point.

(b) Otherwise, if the decimal point follows either the $l$ or the $l+1$ position of the recorded digits, the string is composed of the recorded digits, as well as the character $X$ used as a filler,

(c) Otherwise, the string is created using scientific notation with the character " $E$ ".

3. To the string, append ";".

4. To the string, append the character " $s$ ".

This procedure could be modified in various ways. The first two criteria could be affected by recording fewer digits of $\widehat{\theta}$ or additional digits of $\sigma$. The third criterion could be affected by eliminating the use of the filler $X$ s or replacing the "at" sign with an ampersand.

\section{REFERENCES}

Conway, R., W.L. Maxwell, J.O. McClain and S.L. Worona. (1987), User's Guide to XCELL+ Factory Modeling System, Second edition. Redwood City, California: The Scientific Press.

Goldsman, D.M., B.L. Nelson and B. Schmeiser. (1991), Methods for selecting the best system. Proceedings of the 1991 Winter Simulation Conference, eds. B.L. Nelson, W.D. Kelton, and G.M. Clark, 177-186. Piscataway, New Jersey: The Institute of Electrical and Electronics Engineers.

Schmeiser, B. (1982), Batch size effects in the analysis of simulation output. Operations Research 30, 556-568.

Schmeiser, B. (1992), Modern simulation environments: Statistical issues. Proceedings of the First Industrial Engineering Research Conference, eds. Georgia-Ann Klutke, D.A. Mitta, B.O. Nnaji, and L.M. Seiford, 139-144. Norcross, Georgia: Institute of Industrial Engineers.
Schmeiser, B. (1990), Simulation experiments. Chapter 7 in Handbooks in Operations Research and Management Science, Volume 2: Stochastic Models, D.P. Heyman and M.J. Sobel (eds.), Amsterdam: North-Holland, 295-330.

Schmeiser, B. W., T. Avramidis and S. Hashem. (1990), Overlapping batch statistics. In Proceedings of the Winter Simulation Conference, ed. O. Balci, R. P. Sadowski, and R. E. Nance, 395-398. Piscataway, NJ: IEEE.

Song, W.T. and B. Schmeiser. (1993), Variance of the sample mean: Properties and graphs of quadratic-form estimators. Operations Research 41, May, 501-517.

Song, W.T. and B. W. Schmeiser. (1994), Reporting the precision of simulation experiments. In New Directions in Simulation for Manufacturing and Communications,ed. S. Morito, H. Sakasegawa, K. Yoneda, M. Fushimi, and K. Nakano, 402-407. Tokyo: Operations Research Society of Japan.

Song, W. T. and B. W. Schmeiser. (1995), Optimal meansquared-error batch sizes. Management Science 41: $110-123$.

Song, W. T. and B. W. Schmeiser. (2008). Omitting Meaningless Digits in Point Estimates: The Probability Guarantee of Leading-Digit Rules, Operations Research, forthcoming.

Yoneda, Kiyoshi. (1996), Optimal number of digits to report. Journal of the Operations Research Society of Japan. 39, 3 (September 1996), 424-434.

\section{BIOGRAPHIES}

Wheyming Tina Song is a professor in the Department of Industrial Engineering at the National Tsing Hua University in Taiwan. She received her undergraduate degree in statistics and master's degree in industrial engineering at Cheng-Kung University in Taiwan in 1979. She then received master's degrees in applied mathematics in 1983 and industrial engineering in 1984, both from the University of Pittsburgh. Dr. Song received her Ph.D. from the School of Industrial Engineering at Purdue University in 1989. She joined Tsing Hua in 1990 after spending one year as a visiting assistant professor at Purdue IE. wheyming@ie.nthu.edu.tw

Bruce W. Schmeiser is a professor at Purdue University. He received his Ph.D. from the School of Industrial and Systems Engineering at Georgia Tech in 1975; his undergraduate degree in the Mathematical Sciences and Master's degree in Industrial Engineering are from The University of Iowa. His research interests include stochastic root finding, simulation output analysis, input modeling, random-variate generation, variance-reduction techniques, Markov chain Monte Carlo methods, and applied operations research. He has served in a variety of roles for the IIE, INFORMS and the Winter Simulation Conference. bruce@ecn.purdue.edu 